\title{
Liver inflammation and fibrosis
}

\author{
Yukinori Koyama and David A. Brenner
}

School of Medicine, UCSD, La Jolla, California, USA.

\begin{abstract}
Chronic liver inflammation leads to fibrosis and cirrhosis, which is the 12th leading cause of death in the United States. Hepatocyte steatosis is a component of metabolic syndrome and insulin resistance. Hepatic steatosis may be benign or progress to hepatocyte injury and the initiation of inflammation, which activates immune cells. While Kupffer cells are the resident macrophage in the liver, inflammatory cells such as infiltrating macrophages, T lymphocytes, neutrophils, and DCs all contribute to liver inflammation. The inflammatory cells activate hepatic stellate cells, which are the major source of myofibroblasts in the liver. Here we review the initiation of inflammation in the liver, the liver inflammatory cells, and their crosstalk with myofibroblasts.
\end{abstract}

\section{Introduction}

Chronic liver inflammation leads to liver cirrhosis, which is the 12th leading cause of death in the US (1), accounting for 32,000 deaths in the US and more than 1 million deaths each year worldwide (2). Chronic pathologic processes include viral infection, alcoholic liver disease, nonalcoholic steatohepatitis (NASH), and autoimmune diseases. Depending on the type of underlying liver injury, several mechanisms exist to trigger immune reactions. Chronic immune reactions lead to liver fibrosis. Understanding the mechanism of inflammation and fibrosis is critically important to developing treatments for chronic liver diseases.

Hepatic steatosis is a common consequence of metabolic or toxic stress. This steatosis may progress to hepatic injury in response in alcohol (alcoholic steatohepatitis [ASH]), toxins such as vinyl chloride (toxicant-associated steatohepatitis), chemotherapy (chemotherapy-associated steatohepatitis), or metabolic syndrome with insulin resistance (NASH), which is the most common liver disease in the US $(3,4)$. Injury changes hepatocyte gene expression, resulting in increased expression of TGF- $\beta$, IL-1A, hedgehog ligands, CXCL10, and mesenchymal genes such as twist and snail. The injured steatotic hepatocyte induces inflammation and fibrosis.

Even under physiologic conditions the liver is constantly exposed to exogenous proteins derived from foods, chemicals, drugs, and microbiota in the gut. Immunologically, the liver capillary system is lined with resident macrophages (Kupffer cells) and liver DCs. In pathologic conditions, bone marrow-derived cells, such as infiltrating macrophages, migrate to the liver and work in collaboration with the resident cells. Because of the difficulty in cellular isolation techniques, the inflammatory responses in the liver are not fully understood. In this Review we discuss initiation of inflammation in the liver, inflammatory cells, and their crosstalk with myofibroblasts.

\section{From metabolism to liver injury}

Because the liver plays a central role in metabolism of lipids and glucose, liver inflammation is closely related to metabolic dis-

Conflict of interest: The authors have declared that no conflict of interest exists. Reference information: / Clin Invest. 2017;127(1):55-64. doi:10.1172/JCI88881. orders such as nonalcoholic fatty liver disease (NAFLD), which affects up to $40 \%$ of Western adult populations (3, 4). NAFLD includes a spectrum of diseases ranging from isolated hepatic steatosis to NASH, the progressive form of the disease characterized by inflammation, cellular injury, and fibrosis (which can lead to cirrhosis) that is associated with obesity, type 2 diabetes, hyperlipidemia, and insulin resistance.

Hepatocytes become steatotic as a result of increased de novo lipogenesis, decreased $\beta$ oxidation, and decreased VLDL secretion, potentially resulting in lipotoxicity. Generally, the saturated free fatty acids palmitate and stearate are directly cytotoxic. Furthermore, palmitate can induce the formation of both ceramide and lysophosphatidylcholine (LPC). LPC can activate proapoptotic signaling (5) and lead to extracellular vesicle (EV) release (6). Palmitate-derived C16:0 ceramide is associated with insulin resistance and steatohepatitis (7) and can also induce EV release (Figure 1). EVs, which include exosomes, microvesicles, and apoptotic bodies (8), contain cargoes such as effector proteins and miRNAs that enable cells to transmit signals. TRAIL, CXCL10, and sphingosine-1-phosphate in EVs from injured hepatocytes may be involved in the pathogenesis of NASH through activation or chemotaxis of macrophages (9-11). Additionally, CD4OL and miRNAs (let7f, miR-29a, and miR-340), which have been found in EVs, are known to participate in an alcoholic liver injury mouse model $(12,13)$. Although a pathogenic role for EVs has been demonstrated in cultured cells, it is very difficult to demonstrate an in vivo role for EVs in the pathogenesis of NASH.

The ballooning hepatocytes that characterize NASH are apoptotic cells (14). Liver expression of the death receptor Fas is increased in patients with NAFLD (14). TRAIL-R2 (also known as DR5) mediates apoptosis in response to hepatic lipotoxicity. Treatment of hepatocytes with palmitate increases TRAIL-R2 expression and alters plasma membrane domain organization, resulting in clustering and ligand-independent activation of TRAIL-R2 that leads to caspase 8-dependent cell death (15). In a murine model of Western diet-induced NASH, TRAIL receptor deficiency protected against hepatocyte apoptosis and all other associated pathogenic features of $\mathrm{NASH}$, such as liver injury, inflammation, and fibrosis (16). Pharmacologic inhibition of 


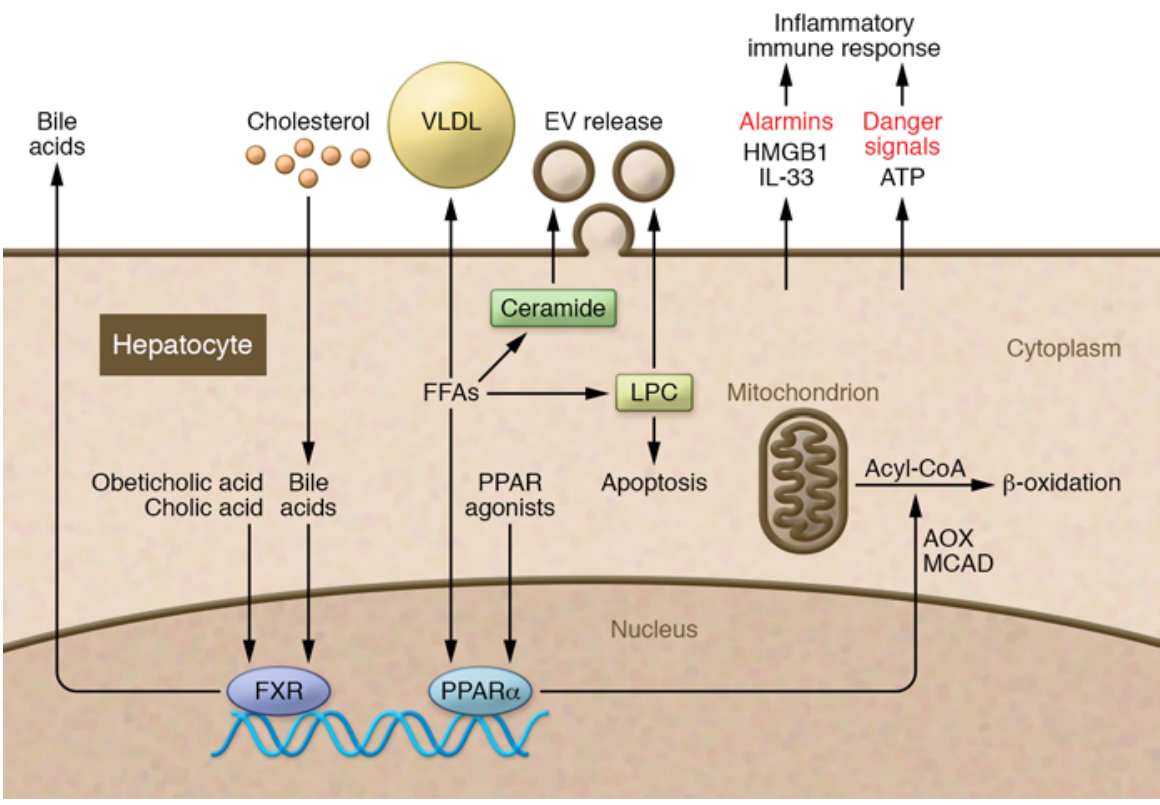

Figure 1. An overview of lipid metabolism in hepatocytes. Hepatocytes become steatotic as a result of increased de novo lipogenesis, decreased $\beta$ oxidation, and decreased VLDL secretion. Free fatty acids (FFAs) such as palmitate can induce the formation of both ceramide and lysophosphatidylcholine (LPC). LPC can activate proapoptotic signaling and lead to extracellular vesicle (EV) release. Palmitate-derived ceramide also induce EV release. HMCB1 and IL-33 are alarmins that are released from hepatocytes in the pathogenesis of chronic liver diseases, including nonalcoholic fatty liver disease. PPAR stimulation facilitates oxidation of lipids by upregulating acyl-CoA-oxidase and MCAD. Thus, PPARs are possible therapeutic targets in nonalcoholic steatohepatitis. FXR signaling facilitates secretion of bile acids and decreases hepatic lipid synthesis and enhances peripheral clearance of VLDL. AOX, acyl-CoA oxidase.

hepatocyte apoptosis or the release of hepatocyte EVs may attenuate liver injury, inflammation, and fibrosis.

Necrosis is considered an accidental process characterized by cell swelling and early loss of plasma membrane integrity, with consequent leakage of proinflammatory mediators. The unregulated nature of necrosis precludes the design of therapeutic interventions for protection of necrosis-associated cell injury and inflammation. Interestingly, necroptosis, an immunogenic form of programmed cell death, is morphologically similar to necrosis and has been implicated in the pathogenesis of inflammationdriven liver disease. TNF- $\alpha$-dependent necroptosis is initiated by the interaction of receptor-interacting proteins 1 and 3 (RIP1/3), which originate in the necrosome, an oligomeric amyloid signaling complex. Necroptosis, as defined by RIP3-dependent mixedlineage kinase domain-like protein activation, is triggered in the liver of mouse models of NASH and in human NAFLD, whereas absence of RIP3 ameliorates liver injury, steatosis, inflammation, and fibrosis in an experimental NASH model (17).

Immune cells are activated by endogenous danger signals known as alarmins (18) or damage-associated molecular patterns (DAMPs), which are released by dead or damaged cells $(19,20)$. The alarmins high-mobility group protein B1 (HMGB1) and IL-33 are released from hepatocytes in chronic liver disease including NAFLD (21-24). ATP and formyl peptide also function as alarmins. ATP released from necrotic cells alerts circulating neutrophils to adhere within liver sinusoids, and formyl-peptide signals released from necrotic cells guide neutrophils into the injury area (25). Furthermore, activation of the NF- $\mathrm{B}$ pathway in injured hepatocytes induces release of a number of proinflammatory cytokines and chemokines such as TNF- $\alpha$, IL-6, and CCL2, which mediate liver inflammation $(26,27)$.

Crosstalk between hepatocytes and immune cells is also mediated by inflammasomes, large multiprotein complexes that sense intracellular danger signals via Nod-like receptors (NLRs) (e.g., NLRP3). NLR forms a complex with the effector molecule pro-caspase- 1 with or without an adapter molecule such as the apoptosisassociated speck-like protein containing a CARD (ASC). Inflammasomes mediate the cleavage and activation of pro-IL-1 $\beta$ and pro-IL-18. Damaged hepatocytes can transfer their danger signals by regulating inflammasome activation in immune cells (Figure 1). In fact, molecules such as ATP and uric acid, which are released from injured hepatocytes, cause inflammasomes in liver Kupffer cells in murine models of ASH and NASH $(28,29)$.

Because the liver is exposed to gutderived microbial products, liver inflammation is modified by the microbiota in the gut. Gut dysbiosis has been demonstrated in obesity $(30,31)$, metabolic syndrome (32), diabetes $(33,34)$, cardiovascular diseases, and NAFLD (35). Boursier and colleagues evaluated gut dysbiosis in NAFLD patients and found that Bacteroides abundance was independently associated with NASH and Ruminococcus with fibrosis (36).

Thus, both endogenous ligands generated from injured cells and exogenous ligands generated from the gut microbiota activate inflammatory pathways.

\section{Inflammatory cells in the liver}

Kupffer cells. Kupffer cells play a central role in liver inflammation. They are resident macrophages that localize within the lumen of the liver sinusoids, accounting for about $30 \%$ of sinusoidal cells (37). In embryogenesis, macrophages migrate into fetal liver from the yolk sac and are detected as $\mathrm{F} 4 / 80^{+}$macrophages at $\mathrm{E} 11$ in mice $(38,39)$. In the late stage of embryonic development and postnatally, these cells proliferate and differentiate into Kupffer cells, which are characterized by peroxidase activity in the nuclear envelope and rough ER (38). Because of their origin, macrophage markers like F4/80, CD11b, and CD68 are also representative markers for Kupffer cells. Although Kupffer cells were previously believed to be unable to self-renew and were instead derived from bone marrowderived monocytes (40-43), recent evidence suggests that Kupffer cells are either a self-renewing population or are derived from local progenitors $(39,44)$. In response to hepatocyte injury, Kupffer cells become activated and express cytokines and signaling molecules. Additionally, activated Kupffer cells display markers of M1-like macrophages or M2-like macrophages depending on the signals 


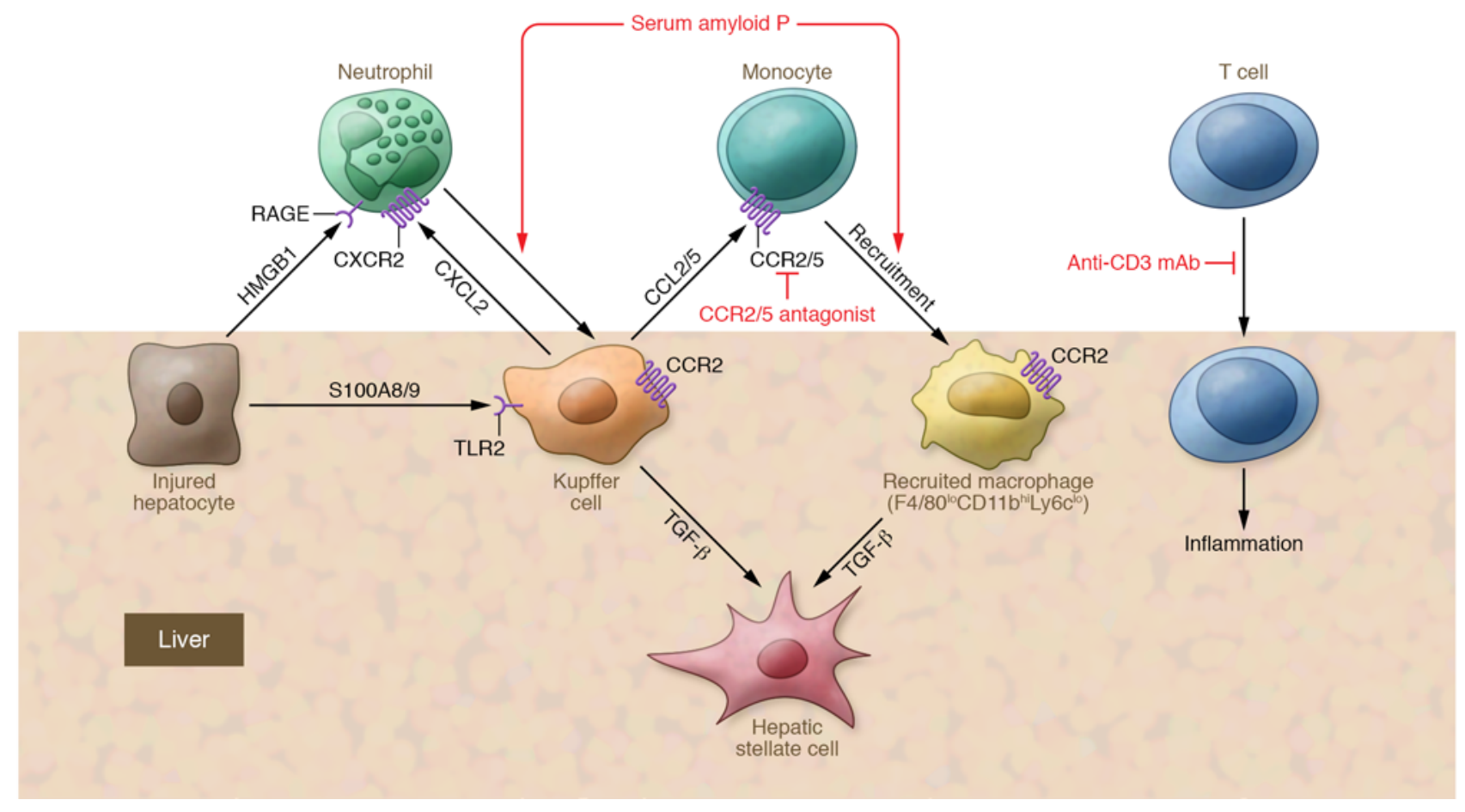

Figure 2. Interaction of immune cells and the liver. The initiation of inflammation results in the trafficking and localization of immune cells to the site of injury, including neutrophils, recruited macrophages, and Tregs. Generation of an intravascular chemokine gradient (e.g., CXCL12) directs neutrophil migration toward damage foci. HMCB1 released by necrotic hepatocytes also mediates the recruitment of neutrophils through interaction with the HMCB1 receptor RACE. Monocyte infiltration into the liver is primarily controlled by CCR2 and its ligand CCL2, which may serve as therapeutic targets in nonalcoholic steatohepatitis (NASH). Serum amyloid P binds to neutrophils and decreases TNF- $\alpha$ - and IL-8-induced neutrophil adhesion to extracellular matrix proteins, attenuates profibrotic macrophages, activates the complement pathway, and promotes phagocytosis of cell debris. Oral administration of an anti-CD3 mAb induces Tregs and has been shown to be effective in a NASH animal model; this antibody is currently in clinical trials. The CCR2/5 antagonist cenicriviroc is now in clinical trials.

they receive from their environment. Inflammation in the liver is regulated by the balance of proinflammatory M1 Kupffer cells and antiinflammatory M2 Kupffer cells (45). Kupffer cells are exposed to various substances such as nutrients and gut-derived bacterial products via the portal circulation and they function to sense and remove pathogens and danger molecules via pattern-recognition receptors (PRRs). PRRs comprise at least two families of sensing proteins: TLRs and NLRs, which detect danger signals including pathogen-associated molecular patterns and alarmins. TLRs recognize gut microbiota-derived bacterial products such as LPS and peptidoglycan. Kupffer cells respond to LPS through TLR4 to produce various inflammatory cytokines including TNF- $\alpha$, IL-1 $\beta$, IL-6, IL-12, IL-18, and chemokines in granulomatous liver disease, ischemia/reperfusion liver injury, NASH, and alcoholic liver disease (46). In turn, activation of many of these mediators worsen insulin resistance and metabolic syndrome.

Recruited macrophages. Recruited bone marrow-derived macrophages are a key component of both acute and chronic liver inflammation and are involved in regression of liver disease. Traditionally, macrophages are divided into proinflammatory (M1), wound-healing (M2), and immunosuppressive (regulatory macrophages) phenotypes (47). M1 macrophages, which are induced by IFN- $\gamma$, LPS, and TNF- $\alpha$ and express proinflammatory cytokines such as TNF- $\alpha$, IL- 6 , and IL-1, are implicated in the pathogenesis of chronic liver inflammation. M2 macrophages, which are induced by IL-4, IL-10, IL-13 and produce IL-10, TGF- $\beta$, PDGF, and EGF, have antiinflammatory effects and promote wound healing (47, 48). In addition to distinct functions and gene expression profiles, M1 and M2 macrophages exhibit distinct metabolic activities (49, 50). M1 macrophages are associated with increased glycolysis and production of NO from arginine by inducible NOS (50). In contrast, M2 macrophages rely on fatty acid oxidation and metabolize arginine through arginase 1 (ARG1) (50). However, extensive transcriptome analysis for recruited macrophages in response to various stimuli revealed a spectrum of macrophage activation between the M1 and M2 poles (51). Thus, the M1/M2 paradigm is probably an oversimplification for hepatic macrophages exposed to various pro- and antiinflammatory stimuli (52). Bone marrowderived macrophages change to Ly $6 c^{\text {lo }}$ population and exhibit a phenotype outside the M1/M2 classification, with increased expression of MMPs, growth factors, and phagocytosis-related genes, including $M m p 9$, Mmp12, Igf1, and the transmembrane glycoprotein NMB (Gpnmb). These macrophages are responsible for tissue remodeling (52). Furthermore, Wang and Kubes described a population of macrophages recruited from peritoneal fluid that is $\mathrm{F} 4 / 80^{\mathrm{hi}} \mathrm{GATA}^{+}$and rapidly infiltrates the injured liver through the mesothelium (53). This invasion was dependent on CD44 and ATP and resulted in rapid replication and switching of macrophages toward an alternatively activated phenotype. Another macrophage recruitment pathway was described by $\mathrm{Xu}$ and col- 
leagues (49), in which NOTCH1 signaling is required for the migration of blood monocytes into the liver and subsequent M1 differentiation. Furthermore, monocyte infiltration into the liver is primarily controlled by CCR2 and its ligand CCL2 (also known as MCP-1) (54-57), providing another potential target for the treatment of NASH. (Figure 2).

Although recruited macrophages and Kupffer cells exhibit similarities, they can be distinguished by several markers. Recruited macrophages are $\mathrm{CD} 11 \mathrm{~b}^{\text {hi }} \mathrm{F} 4 / 80^{\text {lo/int }}$ cells. In contrast, resident Kupffer cells are $\mathrm{CD} 11 \mathrm{~b}^{\mathrm{lo}} \mathrm{F} 4 / 8 \mathrm{O}^{\text {hi }}(58)$. Generally, high expression of CX3CR1 and low expression of the myeloid marker Ly6c characterize patrolling monocytes (59). Kupffer cells are characterized by a lack of CX3CR1 expression (60). Using CX3CR1-GFP transgenic mice, a gene expression analysis of recruited macrophages and Kupffer cells (60) revealed that both Kupffer cells and recruited macrophages highly express TGF- $\beta$, suggesting that both cells contribute to liver fibrogenesis. Further, the infiltrating macrophages are Ly6 $\mathrm{c}^{\text {hi }}$ and differentiate into Ly6 $\mathrm{c}^{\mathrm{lo}}$ macrophages in acetaminophen-induced liver injury (60). Similar transdifferentiation is observed in alcoholic liver injury (58). In NAFLD and alcoholic liver injury, inflammation is predominantly mediated by recruited macrophages, and Kupffer cells exhibit an antiinflammatory M2 phenotype $(45,58)$. Similarly, the increased hepatic macrophage population in diet-induced obesity is from infiltrating bone marrow-derived macrophages (61).

Neutrophils. Neutrophils are rapidly recruited to sites of acute inflammation. Despite the central role of neutrophils in a variety of liver diseases, the molecular mechanisms that allow these cells to home to the liver and their functions within the liver are not well understood. Unlike neutrophil trafficking mechanisms in other organs, the adhesion of neutrophils within the liver is independent of selectins (62). Furthermore, neutrophil recruitment to the infectious/endotoxemic liver is independent of $\alpha 2$ integrin and ICAM-1 crosstalk (63). In one study, a heat-induced liver injury model demonstrated a neutrophil recruitment pathway via the following three steps (25): (a) ATP released by necrotic hepatocytes activated NLRP3 inflammasome via P2X7 receptor signaling; (b) generation of an intravascular chemokine gradient (e.g., CXCL2) directed neutrophil migration toward damage foci (Figure 2); and (c) formyl-peptide signals released from necrotic cells guided neutrophils into the site of injury. Furthermore, the TLR4 ligand S100A9 protein secreted in NASH was a key regulator of hepatic CXCL2 expression and neutrophil recruitment (64). Surprisingly, this study determined that impaired neutrophil recruitment did not aggravate or inhibit liver fibrosis. Another alarmin, HMGB1, which is released by necrotic hepatocytes, also mediates the recruitment of neutrophils in an acetaminophen-induced liver injury model, through interaction with receptor for advanced glycation end products (RAGE) (65). Hyaluronan expressed by the liver sinusoidal endothelial cells (LSECs) interacts with CD44 on the surface of neutrophils, and this interaction plays a critical role in the recruitment of neutrophils to the liver (63). Neutrophil infiltration is associated with inflammation induced by the dietary carbohydrates and cholesterol, whereas the non-metabolic triggers LPS and IL-1 $\beta$ mainly induce intrahepatic accumulation of mononuclear cells, suggesting that different inducers may elicit different inflammatory responses (66). It has been suggested that increased myeloperoxidase secreted by neutrophils may cause oxidative damage to hepatocytes and contribute to the development of NASH $(67,68)$. Additionally, high neutrophil elastase activity and low $\alpha-1$ antitrypsin determine the severity of experimental NASH (69). Thus, there are several lines of evidence that neutrophils contribute to the metabolic syndrome and, in particular, to the pathogenesis of NASH.

DCs. DCs are professional antigen-presenting cells that capture and process antigens, migrate to lymphoid organs, and secrete cytokines to initiate both innate and adaptive immune responses. However, liver DCs have tolerogenic functions, including induction of Tregs and deletion of active T cells (70), thereby minimizing autoimmune reactions. DCs are divided into classical type 1 DCs (cDC1), cDC2, and plasmacytoid DCs (pDCs), and their markers have been identified in both mice and humans $(71,72)$. In humans and mice, the cDC2 cells are most abundant in the liver and they exhibit tolerogenic capacity. pDCs secrete type I IFNs during viral infections (73), while cDC1s present antigens to T cells. After liver injury, DCs gain the capacity to induce hepatic stellate cells, NK cells, and T cells to mediate inflammation (74). DCs are also involved in the regression of fibrosis after liver injury through their production of MMP9 (75). Although the role of DC migration in liver pathology has not been explored in detail, extrahepatic DCs affect liver inflammation in the context of NAFLD pathogenesis. Bertola and colleagues found that CD11 $\mathrm{c}^{+}$DCs in adipose tissue control inflammation by regulating the switch toward Th17 cell responses in obesity-associated insulin resistance in both obese patients and experimental murine models (76).

$T$ lymphocytes. Unlike other organs, inflammation in the liver is mainly sterile (non-infectious), excluding parasite-induced fibrosis. Therefore, "innate-like" mechanisms (Th17, NK cells, NKT cells, MAIT cells, innate lymphoid cells) are major contributors to liver inflammation.

Th17 cells are proinflammatory cells that produce IL-17 cytokines, which have been implicated in the promotion of liver inflammation and fibrosis (77-79). Based on evidence from mouse models, IL-17 has a strong profibrogenic effect through two independent mechanisms (79). First, IL-17 stimulates Kupffer cells and macrophages to express inflammatory cytokines IL-6, IL-1 $\beta$, and TNF- $\alpha$, as well as the major fibrogenic cytokine TGF- $\beta 1$. Second, IL-17 directly stimulates hepatic stellate cells to express collagen type I and promotes their differentiation into fibrogenic myofibroblasts via STAT3. Levels of IL-17A are elevated in serum of patients with alcoholic liver disease, chronic hepatitis $\mathrm{B}$ and chronic hepatitis $\mathrm{C}$ and are correlated with increased numbers of circulating Th17 cells and histologic manifestations of liver fibrosis $(77,78)$. IL-17-expressing cells, including Th17 cells, have been linked to progressive NAFLD (80). Anti-IL-17 biological therapies are currently in clinical trials for autoimmune inflammatory diseases and may be applicable in liver inflammation and fibrosis (81).

Mucosal-associated invariant T (MAIT) cells are innatelike $\mathrm{T}$ cell populations characterized by the invariant TCR chain $\mathrm{V} \alpha 7.2-\mathrm{J} \alpha 33 / \mathrm{J} \alpha 20 / \mathrm{J} \alpha 12$ in humans and V $\alpha 19-\mathrm{J} \alpha 33$ in mice (82). MAIT cells are highly enriched in the human liver, representing $20 \%$ to $50 \%$ of intrahepatic T cells (83). MAIT cells reside in peri-biliary areas of portal tracts in healthy adults, but are reduced in blood and liver in patients with autoimmune hepa- 
titis, primary biliary cholangitis, primary sclerosing cholangitis, alcoholic liver disease and NASH (84). A recent study of MAIT cells in obese individuals and patients with type 2 diabetes demonstrated a marked reduction in circulating MAIT cells in both patient groups compared with controls (85). MAIT cells, which are prominent producers of IL-17, were enriched in adipose tissue compared with the peripheral blood of obese patients. A direct role of MAIT cells in NASH has not been demonstrated but will be an important area for future exploration.

Unlike in humans, mouse liver is enriched in NKT cells, which can constitute up to $10 \%$ of the leukocyte fraction in the mouse liver (or up to $40 \%$ of the hepatic lymphocytes) (86). NKT cells can recognize both exogenous and endogenous lipid antigens in the context of the major histocompatibility complex-like molecule CD1d. NKT cells are classified into two main subsets; type I or invariant NKT cells and type II or diverse NKT cells. Type I NKT cells are activated by glycolipids such as $\alpha \mathrm{GalCer}$ and TLR-mediated signaling and/or by cytokines (IL-12, IL-18, or type I IFN). Murine alcoholic liver injury models exhibit an increased number of activated type I NKT cells, but not type II cells, in the liver (87). Type II NKT cells have protective roles in alcoholic liver injury models (87).

Tregs are antiinflammatory regulators of obesity-related metabolic dysregulation (88). Tregs have been found to be reduced in the blood of obese individuals (89). Accordingly, Tregs have been studied in the context of NASH pathogenesis. CD28 and B7 costimulation is critical in promoting the development of Tregs from naïve T cells (90) and CD28 KO mice display attenuated fat accumulation and inflammation, probably due to a defect in Tregs (91). Further, antibody-mediated B7 blockade improves steatohepatitis in mice (92). Treg modulation may provide a novel therapeutic strategy in NASH (Figure 2).

\section{From liver inflammation to fibrosis}

Hepatocyte injury followed by inflammation and activation of the innate immune system leads to liver fibrosis mediated by hepatic stellate cell activation and extracellular matrix (ECM) secretion and deposition. Quiescent hepatic stellate cells (qHSCs) are located in the space of Disse, a perisinusoidal space between hepatocytes and LSECs. qHSCs store vitamin A in lipid droplets, which represent the major vitamin A storage site for humans (93). qHSCs express adipogenic transcription factors such as $\operatorname{PPAR} \gamma$, adiponectin receptor 1 , perilipin 2 (PLIN2/ADFP), $\mathrm{C} / \mathrm{EBP} \delta, \mathrm{C} / \mathrm{EBP} \alpha, \mathrm{C} / \mathrm{EBP} \beta$, and SREBP-1c $(94,95)$. Hepatic stellate cell differentiation into myofibroblasts is characterized by decreased vitamin A with loss of the adipogenic phenotype (94, $96,97)$. Activated hepatic stellate cells (aHSCs/myofibroblasts) are the major source of ECM production in the liver fibrosis induced by NASH and also contribute to liver inflammation by secreting cytokines.

Hepatic stellate cell activation results from the inflammatory activity of liver immune cells, predominantly macrophages. Macrophage-derived TGF- $\beta 1$ activates hepatic stellate cells and is the most potent known fibrogenic agonist (98). Hepatic macrophages, including Kupffer cells and recruited macrophages, also enhance liver fibrosis by promoting the survival of aHSCs in a NF-אB-dependent manner (99). Macrophage/hepatic stellate cell crosstalk is mediated by IL- 1 and TNF- $\alpha$. Neutralization of these cytokines in co-culture experiments or genetic ablation of their receptors in mouse models led to decreased fibrosis and increased apoptosis of hepatic stellate cells (99). Consistently, inhibition of NF- $\kappa$ B with sulfasalazine stimulated apoptosis of activated rat and human hepatic stellate cells, impairing hepatic fibrosis (100). Furthermore, in NASH, DAMPs from injured hepatocytes activate the inflammasome in Kupffer cells, resulting in release of IL-1 $\beta$, thereby inducing hepatic stellate cell activation (101).

In obesity, hepatic stellate cell activation is also mediated by leptin, an adipocyte-derived hormone (102). Leptin receptordeficient rats are protected from $\mathrm{CCl}_{4}$-induced liver fibrosis (103). Furthermore, leptin stimulation leads to TGF- $\beta 1$ expression in Kupffer cells and conditioned medium of leptin-stimulated Kupffer cells activates hepatic stellate cells (104). Leptin also maintains the activated phenotype of hepatic stellate cells via the upregulation of the hedgehog pathway (105). In contrast, the pro-adipogenic factor PPAR $\gamma$ abrogates the activation of hepatic stellate cells (106).

ROS are generated by various liver injuries such as alcohol abuse, hepatitis virus infection and chronic cholestasis and contribute to hepatic fibrogenesis. ROS stimulates the production of collagen I in aHSCs/myofibroblasts, acting as an intracellular signaling mediator for TGF- $\beta 1$-induced fibrosis (107). The multicomponent NADPH oxidase (NOX) complexes and the mitochondrial respiratory pathway are the two major producers of endogenous ROS. NOX play an important role in liver fibrogenesis. Among the seven members of the NOX family (NOX 1-5; DUOX1 and -2), NOX1, NOX2, and NOX4 are expressed in the liver, and specifically in aHSCs/myofibroblasts (108). NOX2 induces the oxidative burst required by neutrophils to kill pathogenic bacteria (109), while NOX1 and NOX4 are expressed in hepatocytes, Kupffer cells, and hepatic stellate cells (108). NOX1 is catalytically activated by pro-fibrogenic agonists including angiotensin II (Ang II), LPS, and PDGF, which induce hepatic stellate cell activation and proliferation (108). NOX4 is a TGFresponsive gene and is regulated at the level of transcription (110). NOX1- or NOX4-deficient hepatic stellate cells produce less ROS in response to Ang II compared with cells that express these enzymes (111). Both NOX1 KO and NOX4 KO mice exhibit attenuated liver fibrosis mediated by decreased hepatic stellate cell activation. Importantly, NOX1 and NOX4 are elevated in mouse models of NASH, and NOX inhibition decreases inflammation and fibrosis in NASH models (112).

TLR4 activation can lead to ROS signaling, and ROS may modulate signaling downstream of TLR4 via NOX activation. Kupffer cells and macrophages are targets for the TLR4 ligand LPS, and these cells express profibrogenic cytokines that activate hepatic stellate cells $(46)$, which also express TLR4 $(113,114)$. Several lines of evidence indicate that LPS/TLR4 signaling provides a mechanistic link between inflammation and fibrosis $(114,115)$. In response to LPS, hepatic stellate cells express chemokines (MCP-1, MIP-1 $\alpha$, MIP-1 $\beta$, RANTES, KC, MIP-2, and IP-10) and adhesion molecules (ICAM-1, VCAM-1, and E-selectin) to recruit Kupffer cells and circulating macrophages (114). LPS also enhances TGF- $\beta$ signaling in hepatic stellate cells via down regulation of bambi, a TGF- $\beta$ pseudo receptor that drives hepatic fibrosis through activation of hepatic stellate cells (refs. 111, 114, 116, and Figure 1). 


\section{No fibrosis without inflammation?}

In most liver injury diseases such as NASH, chronic liver inflammation precedes liver fibrosis. Various liver fibrosis models in rodents also evoke inflammation. On the other hand, overexpression of PDGF-B in hepatocytes causes liver fibrosis with minimal liver inflammation (117, 118). Additionally, hemochromatosis is characterized by liver cirrhosis with minimal inflammation (119), and the murine hemochromatosis model also shows minimal inflammation with massive fibrosis. In iron overload, ferritin released from damaged hepatocytes contributes to the local hepatic ferritin concentration. Tissuederived ferritin has been demonstrated to act as a proinflammatory cytokine in hepatic stellate cell biology by inducing iron-independent, $\mathrm{PKC} / \mathrm{NF}-\mathrm{kB}$ transcriptional regulation of inflammatory genes that are associated with the activation of hepatic stellate cells (120). aHSCs/myofibroblasts also express a specific transferrin receptor that mediates enhanced expression of $\alpha$-SMA and collagen- $\alpha 1$ (121). A murine model of hereditary hemochromatosis ( $\mathrm{HH} ; \mathrm{HFe} \mathrm{KO}$ mice) exhibits attenuated inflammatory responses to Salmonella infection and LPS induction (118). In $\mathrm{Hfe}^{-/-}$macrophages, LPS-induced TNF- $\alpha$ and IL-6 secretion was attenuated (118). These results suggest a role of iron in the regulation of the inflammatory response leading to liver fibrosis. However, about half of patients with $\mathrm{HH}$ show histologic evidence of portal inflammation. Hepatic venous outflow obstruction also causes liver fibrosis without massive liver inflammation. Right heart failure (cardiac hepatopathy) and Budd-Chiari syndrome (thrombosis of the hepatic veins) are the representative pathology. Further, outflow obstruction histology shows minimal inflammation in the liver (122). These findings suggest that unlike idiopathic pulmonary fibrosis (123), the pathophysiology of the "crypt-inflammatory" liver fibrosis is more a product of dysregulated inflammation than of fibroblast dysfunction. Finally, advanced stages of NASH may have less inflammation than earlier stages, and the NASH activity score does not correlate with the stage of fibrosis. Thus, NASH may progress from an inflammatory, steatotic early phase driven by metabolic syndrome to a predominantly fibrotic later stage, where fibrogenic stimuli such as TGF- $\beta$ perpetuate scarring.

\section{Perspectives}

NASH and NAFLD are characterized by decreased consumption and secretion of lipids. Therefore, enhancement of lipid secretion and consumption may be a promising therapeutic strategy for NAFLD. PPAR stimulation facilitates oxidation of lipids by upregulating acyl-CoA oxidase and medium-chain acyl-CoA dehydrogenase (MCAD). Thus, PPARs are possible therapeutic targets in NASH (Figure 1). In fact, PPAR agonists have been reported and are now in clinical trials for the treatment of NASH $(124,125)$. FXR signaling is also a potential target because it facilitates secretion of bile acids (126), resulting in decreased hepatic storage of triglycerides and fatty acid esters (Figure 1). Moreover, FXR signaling caused decreased hepatic lipid synthesis and enhanced peripheral clearance of VLDL. As a result, it promotes insulin sensitivity, decreases hepatic gluconeogenesis and circulating triglycerides (127). Based on these metabolic effects, pharmacologic activation of FXR has been proposed as a target for the treatment of NASH. Cholic acids and obeticholic acid are reported as agonists of FXR, and both are now in clinical trials (refs. 128-131, and Figure 1).

CCR2 and CCR5 and their ligands CCL2 and CCL5 are implicated in the pathogenesis of liver inflammation and fibrosis, especially in NASH. In response to hepatocyte injury, Kupffer cells secrete CCL2, which recruits monocytes to the liver. Inhibition of CCL2/CCR2 or CCL5/CCR5 has been shown to attenuate liver fibrosis in mice $(56,57,132-134)$. The CCR2/5 antagonist cenicriviroc is now in a phase 2 clinical trial (135) (Figure 2).

New targets for treating chronic liver diseases with fibrosis can be analyzed in the context of recent insights into the mechanisms of liver inflammation. Serum amyloid P (SAP; also known as pentraxin-2) is a $27-\mathrm{kDa}$ protein that is produced by the liver and secreted into the blood and circulates as stable 135-kDa pentamers (136). SAP binds to neutrophils and decreases TNF- $\alpha-$ and IL-8-induced neutrophil adhesion to ECM proteins, attenuates profibrotic macrophages, activates the complement pathway, and promotes phagocytosis of cell debris (136-139).

After activation, Kupffer cells and recruited macrophages produce TGF- $\beta 1$, which induces differentiation of hepatic stellate cells into myofibroblasts. Local suppression of TGF- $\beta 1$ activity remains a major challenge of antifibrotic therapy. Neutralization of TGF- $\beta$ in animal models inhibits liver fibrosis and reduces the risk of developing cholangiocarcinoma (140). Fresolimumab (GC1008), a human anti-TGF- $\beta 1 \mathrm{mAb}$ that neutralizes all isoforms of TGF- $\beta$, has demonstrated an acceptable safety profile and preliminary evidence of antitumor activity in patients with advanced malignant melanoma and renal cell carcinoma (141-143). Fresolimumab also exhibits antiinflammatory and anti-fibrotic effects in systemic sclerosis patients (144), suggesting that therapeutic targeting of TGF- $\beta 1$ is a potential strategy in fibrotic liver disease.

Because oxidative stress mediates hepatocyte death and hepatic stellate cell activation, regulation of ROS is a promising strategy for liver fibrosis therapy (145). PPAR $\delta$ is a key metabolic regulator with pleiotropic actions in various tissues including fat, skeletal muscle, and liver. PPAR $\delta$ agonists protect hepatocytes from cell death by reducing ROS generation in hepatocytes, leading to decreased liver fibrosis (146).

The cellular membrane component phosphatidylcholine (PC) is generated by a transmethylation reaction from phosphatidylethanolamine (PE) via a metabolic pathway that utilizes Sadenosylmethionine (SAMe) as a methyl donor (147). The PC/PE ratio may be a key regulator of cell membrane integrity and play a role in the progression of steatosis to NASH (148). Furthermore, the formation of $\mathrm{PC}$ is reduced in various chronic liver diseases including intrahepatic cholestasis, cholestasis of pregnancy, and alcoholic liver disease (149-151). There have been few randomized controlled trials to assess the efficacy of SAMe in chronic liver diseases $(152,153)$, but in studies conducted thus far, SAMe treatment improved pruritus.

Tregs are important for the pathogenesis of NAFLD and NASH. Therefore, enhancement of Treg function may be a therapeutic strategy for the treatment of NASH. Oral administration of an anti-CD3 mAb induces Tregs and has been shown to be effective in a NASH animal model; this antibody is currently in clinical trials (refs. 154, 155, and Figure 2). 
In conclusion, the cellular and molecular mechanisms of liver inflammation and fibrosis are being investigated extensively. Liver inflammation and fibrogenesis are controlled by complex immunologic pathways that implicate many possible therapeutic targets. Enhancement of our understanding of the mechanisms underlying inflammation should be translated into clinical therapies.

\section{Acknowledgments}

This article is supported by NIH grants (to DAB) AA011999, ES010337, and AA021856.

Address correspondence to: David A. Brenner, 9500 Gilman Drive, \#0063, La Jolla, California 92093, USA. Phone: 858.822.5339; E-mail:dbrenner@ucsd.edu.
1. Centers for Disease Control and Prevention. Health, United States, 2015 Web Site. CDC Web site. http://www.cdc.gov/nchs/hus/contents2015.htm\#017. Updated April 27, 2016. Accessed November 11, 2016.

2. Mokdad AA, et al. Liver cirrhosis mortality in 187 countries between 1980 and 2010: a systematic analysis. BMC Med. 2014;12:145.

3. Sayiner M, Koenig A, Henry L, Younossi ZM. Epidemiology of nonalcoholic fatty liver disease and nonalcoholic steatohepatitis in the United States and the rest of the world. Clin Liver Dis. 2016;20(2):205-214.

4. Satapathy SK, Sanyal AJ. Epidemiology and natural history of nonalcoholic fatty liver disease. Semin Liver Dis. 2015;35(3):221-235.

5. Kakisaka K, et al. Mechanisms of lysophosphatidylcholine-induced hepatocyte lipoapoptosis. Am J Physiol Gastrointest Liver Physiol. 2012;302(1):G77-G84.

6. Ibrahim SH, et al. Mixed lineage kinase 3 deficient mice are protected against the high fat high carbohydrate diet-induced steatohepatitis. Liver Int. 2014;34(3):427-437.

7. Turpin SM, et al. Obesity-induced CerS6dependent $\mathrm{C} 16: 0$ ceramide production promotes weight gain and glucose intolerance. Cell Metab. 2014;20(4):678-686.

8. Hirsova P, et al. Extracellular vesicles in liver pathobiology: small particles with big impact [published online ahead of print September 15, 2016]. Hepatology. doi:10.1002/hep.28814.

9. Ibrahim SH, et al. Mixed lineage kinase 3 mediates release of C-X-C motif ligand 10-bearing chemotactic extracellular vesicles from lipotoxic hepatocytes. Hepatology. 2016;63(3):731-744.

10. Kakazu E, Mauer AS, Yin M, Malhi H. Hepatocytes release ceramide-enriched pro-inflammatory extracellular vesicles in an IRE1 $\alpha$-dependent manner. J Lipid Res. 2016;57(2):233-245.

11. Hirsova P, et al. Lipid-induced signaling causes release of inflammatory extracellular vesicles from hepatocytes. Gastroenterology. 2016;150(4):956-967.

12. Eguchi A, et al. Extracellular vesicles released by hepatocytes from gastric infusion model of ALD contain a miRNA barcode that can be detected in blood [published online ahead of print September 17, 2016]. Hepatology. doi:10.1002/hep.28838.

13. Verma VK, et al. Alcohol stimulates macrophage activation through caspase-dependent hepatocyte derived release of $\mathrm{CD} 4 \mathrm{OL}$ containing extracellular vesicles. J Hepatol. 2016;64(3):651-660.

14. Feldstein AE, et al. Hepatocyte apoptosis and fas expression are prominent features of human nonalcoholic steatohepatitis. Gastroenterology. 2003;125(2):437-443.
15. Cazanave SC, et al. Death receptor 5 signaling promotes hepatocyte lipoapoptosis. J Biol Chem. 2011;286(45):39336-39348.

16. Idrissova $L$, et al. TRAIL receptor deletion in mice suppresses the inflammation of nutrient excess. JHepatol. 2015;62(5):1156-1163.

17. Afonso MB, et al. Necroptosis is a key pathogenic event in human and experimental murine models of non-alcoholic steatohepatitis. Clin Sci. 2015;129(8):721-739.

18. Oppenheim JJ, Yang D. Alarmins: chemotactic activators of immune responses. Curr Opin Immunol. 2005;17(4):359-365.

19. Harris HE, Raucci A. Alarmin(g) news about danger: workshop on innate danger signals and HMGB1.EMBO Rep. 2006;7(8):774-778.

20. Rittirsch D, Flierl MA, Ward PA. Harmful molecular mechanisms in sepsis. Nat Rev Immunol. 2008;8(10):776-787.

21. Sass G, Heinlein S, Agli A, Bang R, Schümann J, Tiegs G. Cytokine expression in three mouse models of experimental hepatitis. Cytokine. 2002;19(3):115-120.

22. Marvie P, et al. Interleukin-33 overexpression is associated with liver fibrosis in mice and humans. J Cell Mol Med. 2010;14(6B):1726-1739.

23. Arshad MI, et al. NKT cells are required to induce high IL-33 expression in hepatocytes during ConA-induced acute hepatitis. Eur Jimmunol. 2011;41(8):2341-2348.

24. Li L, et al. Nuclear factor high-mobility group box1 mediating the activation of Toll-like receptor 4 signaling in hepatocytes in the early stage of nonalcoholic fatty liver disease in mice. Hepatology. 2011;54(5):1620-1630.

25. McDonald B, et al. Intravascular danger signals guide neutrophils to sites of sterile inflammation. Science. 2010;330(6002):362-366.

26. Son G, Iimuro Y, Seki E, Hirano T, Kaneda Y, Fujimoto J. Selective inactivation of NF- $\mathrm{kB}$ in the liver using NF-kB decoy suppresses CCl4-induced liver injury and fibrosis. Am JPhysiol Gastrointest Liver Physiol. 2007;293(3):G631-G639.

27. Zhang B, et al. Neurotropin suppresses inflammatory cytokine expression and cell death through suppression of NF- $\mathrm{KB}$ and JNK in hepatocytes. PLoS One. 2014;9(12):e114071.

28. Petrasek J, et al. Metabolic danger signals, uric acid and ATP, mediate inflammatory cross-talk between hepatocytes and immune cells in alcoholic liver disease. J Leukoc Biol. 2015;98(2):249-256.

29. Ganz M, et al. Progression of non-alcoholic steatosis to steatohepatitis and fibrosis parallels cumulative accumulation of danger signals that promote inflammation and liver tumors in a high fat-cholesterol-sugar diet model in mice. J Transl Med. 2015;13:193.
30. Turnbaugh PJ, et al. A core gut microbiome in obese and lean twins. Nature. 2009;457(7228):480-484.

31. Turnbaugh PJ, Ley RE, Mahowald MA, Magrini V, Mardis ER, Gordon JI. An obesity-associated gut microbiome with increased capacity for energy harvest. Nature. 2006;444(7122):1027-1031.

32. Murphy EF, et al. Divergent metabolic outcomes arising from targeted manipulation of the gut microbiota in diet-induced obesity. Gut . 2013;62(2):220-226.

33. Qin J, et al. A metagenome-wide association study of gut microbiota in type 2 diabetes. Nature. 2012;490(7418):55-60.

34. Vrieze A, et al. Transfer of intestinal microbiota from lean donors increases insulin sensitivity in individuals with metabolic syndrome. Gastroenterology. 2012;143(4):913-6.e7.

35. Schnabl B, Brenner DA. Interactions between the intestinal microbiome and liver diseases. Gastroenterology. 2014;146(6):1513-1524.

36. Boursier J, et al. The severity of nonalcoholic fatty liver disease is associated with gut dysbiosis and shift in the metabolic function of the gut microbiota. Hepatology. 2016;63(3):764-775.

37. Bouwens L, Baekeland M, De Zanger R, Wisse E. Quantitation, tissue distribution and proliferation kinetics of Kupffer cells in normal rat liver. Hepatology. 1986;6(4):718-722.

38. Naito M, Yamamura F, Nishikawa S, Takahashi K. Development, differentiation, and maturation of fetal mouse yolk sac macrophages in cultures. JLeukoc Biol. 1989;46(1):1-10.

39. Gomez Perdiguero E, et al. Tissue-resident macrophages originate from yolk-sacderived erythro-myeloid progenitors. Nature. 2015;518(7540):547-551.

40. Diesselhoff-den Dulk MM, Crofton RW, van Furth R. Origin and kinetics of Kupffer cells during an acute inflammatory response. Immunology. 1979;37(1):7-14.

41. Scott CL, et al. Bone marrow-derived monocytes give rise to self-renewing and fully differentiated Kupffer cells. Nat Commun. 2016;7:10321.

42. Hoeffel G, et al. Adult Langerhans cells derive predominantly from embryonic fetal liver monocytes with a minor contribution of yolk sac-derived macrophages. J Exp Med. 2012;209(6):1167-1181.

43. Sheng J, Ruedl C, Karjalainen K. Most tissue-resident macrophages except microglia are derived from fetal hematopoietic stem cells. Immunity. 2015;43(2):382-393.

44. Schulz C, et al. A lineage of myeloid cells independent of Myb and hematopoietic stem cells. Science. 2012;336(6077):86-90.

45. Wan J, et al. M2 Kupffer cells promote M1 Kupffer cell apoptosis: a protective mechanism against 
alcoholic and nonalcoholic fatty liver disease. Hepatology. 2014;59(1):130-142.

46. Seki E, et al. Lipopolysaccharide-induced IL-18 secretion from murine Kupffer cells independently of myeloid differentiation factor 88 that is critically involved in induction of production of IL-12 and IL-1beta. J Immunol. 2001;166(4):2651-2657.

47. Mosser DM, Edwards JP. Exploring the full spectrum of macrophage activation. Nat Rev Immunol. 2008;8(12):958-969.

48. Zhang F, et al. TGF- $\beta$ induces M2-like macrophage polarization via SNAIL-mediated suppression of a pro-inflammatory phenotype [published online ahead of print July 13, 2016]. Oncotarget. doi:10.18632/oncotarget.10561.

49. Xu J, et al. NOTCH reprograms mitochondrial metabolism for proinflammatory macrophage activation. JClin Invest. 2015;125(4):1579-1590.

50. Munder M, Eichmann K, Modolell M. Alternative metabolic states in murine macrophages reflected by the nitric oxide synthase/arginase balance: competitive regulation by $\mathrm{CD} 4^{+} \mathrm{T}$ cells correlates with Th1/Th2 phenotype. J Immunol. 1998;160(11):5347-5354.

51. Xue J, et al. Transcriptome-based network analysis reveals a spectrum model of human macrophage activation. Immunity. 2014;40(2):274-288.

52. Ramachandran P, et al. Differential Ly-6C expression identifies the recruited macrophage phenotype, which orchestrates the regression of murine liver fibrosis. Proc Natl Acad Sci U S A. 2012;109(46):E3186-E3195.

53. Wang J, Kubes P. A reservoir of mature cavity macrophages that can rapidly invade visceral organs to affect tissue repair. Cell. 2016;165(3):668-678.

54. Karlmark KR, et al. Hepatic recruitment of the inflammatory $\mathrm{Gr}^{+}$monocyte subset upon liver injury promotes hepatic fibrosis. Hepatology. 2009;50(1):261-274.

55. Zimmermann HW, et al. Functional contribution of elevated circulating and hepatic non-classical CD14CD16 monocytes to inflammation and human liver fibrosis. PLoS One. 2010;5(6):e11049.

56. Seki E, et al. CCR2 promotes hepatic fibrosis in mice. Hepatology. 2009;50(1):185-197.

57. Mitchell C, et al. Dual role of CCR2 in the constitution and the resolution of liver fibrosis in mice. Am J Pathol. 2009;174(5):1766-1775.

58. Wang M, You Q, Lor K, Chen F, Gao B, Ju C. Chronic alcohol ingestion modulates hepatic macrophage populations and functions in mice. J Leukoc Biol. 2014;96(4):657-665.

59. Geissmann F, Manz MG, Jung S, Sieweke MH, Merad M, Ley K. Development of monocytes, macrophages, and dendritic cells. Science. 2010;327(5966):656-661.

60. Zigmond E, et al. Infiltrating monocyte-derived macrophages and resident kupffer cells display different ontogeny and functions in acute liver injury. J Immunol. 2014;193(1):344-353.

61. Stefanovic-Racic M, et al. Dendritic cells promote macrophage infiltration and comprise a substantial proportion of obesity-associated increases in $\mathrm{CD} 11 \mathrm{c}^{+}$cells in adipose tissue and liver. Diabetes. 2012;61(9):2330-2339.

62. Wong J, et al. A minimal role for selectins in the recruitment of leukocytes into the inflamed liver microvasculature. J Clin Invest. 1997;99(11):2782-2790.

63. McDonald B, et al. Interaction of CD44 and hyaluronan is the dominant mechanism for neutrophil sequestration in inflamed liver sinusoids. JExp Med. 2008;205(4):915-927.

64. Moles A, et al. A TLR2/S100A9/CXCL-2 signaling network is necessary for neutrophil recruitment in acute and chronic liver injury in the mouse. J Hepatol. 2014;60(4):782-791.

65. Huebener P, et al. The HMGB1/RAGE axis triggers neutrophil-mediated injury amplification following necrosis. JClin Invest. 2015;125(2):539-550.

66. Liang $\mathrm{W}$, et al. Metabolically induced liver inflammation leads to NASH and differs from LPS- or IL-1 $\beta$-induced chronic inflammation. Lab Invest. 2014;94(5):491-502.

67. Rensen SS, et al. Increased hepatic myeloperoxidase activity in obese subjects with nonalcoholic steatohepatitis. Am J Pathol. 2009;175(4):1473-1482.

68. Nijhuis J, Rensen SS, Slaats Y, van Dielen FM, Buurman WA, Greve JW. Neutrophil activation in morbid obesity, chronic activation of acute inflammation. Obesity (Silver Spring). 2009;17(11):2014-2018.

69. Zang S, et al. Increased ratio of neutrophil elastase to $\alpha 1$-antitrypsin is closely associated with liver inflammation in patients with nonalcoholic steatohepatitis. Clin Exp Pharmacol Physiol. 2016;43(1):13-21.

70. Goubier A, et al. Plasmacytoid dendritic cells mediate oral tolerance. Immunity. 2008;29(3):464-475.

71. Eckert C, Klein N, Kornek M, Lukacs-Kornek V. The complex myeloid network of the liver with diverse functional capacity at steady state and in inflammation. Front Immunol. 2015;6:179.

72. Heymann F, Tacke F. Immunology in the liver-from homeostasis to disease. Nat Rev Gastroenterol Hepatol. 2016;13(2):88-110.

73. O'Keeffe M, et al. Mouse plasmacytoid cells: long-lived cells, heterogeneous in surface phenotype and function, that differentiate into CD8(+) dendritic cells only after microbial stimulus. J Exp Med. 2002;196(10):1307-1319.

74. Tokita D, et al. Poor allostimulatory function of liver plasmacytoid DC is associated with proapoptotic activity, dependent on regulatory $\mathrm{T}$ cells. J Hepatol. 2008;49(6):1008-1018.

75. Jiao J, et al. Dendritic cell regulation of carbon tetrachloride-induced murine liver fibrosis regression. Hepatology. 2012;55(1):244-255.

76. Bertola A, et al. Identification of adipose tissue dendritic cells correlated with obesityassociated insulin-resistance and inducing Th17 responses in mice and patients. Diabetes. 2012;61(9):2238-2247.

77. Lemmers A, et al. The interleukin-17 pathway is involved in human alcoholic liver disease. Hepatology. 2009;49(2):646-657.

78. Ge J, Wang K, Meng QH, Qi ZX, Meng FL, Fan YC. Implication of Th17 and Th1 cells in patients with chronic active hepatitis B. J Clin Immunol. 2010;30(1):60-67.

79. Meng F, et al. Interleukin-17 signaling in inflam- matory, Kupffer cells, and hepatic stellate cells exacerbates liver fibrosis in mice. Gastroenterology. 2012;143(3):765-776.e1.

80. Rau M, et al. Progression from nonalcoholic fatty liver to nonalcoholic steatohepatitis is marked by a higher frequency of Th17 cells in the liver and an increased $\mathrm{Th} 17 /$ resting regulatory $\mathrm{T}$ cell ratio in peripheral blood and in the liver. J Immunol. 2016;196(1):97-105.

81. Hueber W, et al. Effects of AIN457, a fully human antibody to interleukin-17A, on psoriasis, rheumatoid arthritis, and uveitis. Sci Transl Med. 2010;2(52):52ra72.

82. Kurioka A, Walker LJ, Klenerman P, Willberg CB. MAIT cells: new guardians of the liver. Clin Transl Immunology. 2016;5(8):e98.

83. Dusseaux M, et al. Human MAIT cells are xenobiotic-resistant, tissue-targeted, CD161 ${ }^{\mathrm{hi}}$ IL-17secreting T cells. Blood. 2011;117(4):1250-1259.

84. Jeffery HC, et al. Biliary epithelium and liver $B$ cells exposed to bacteria activate intrahepatic MAIT cells through MR1. J Hepatol. 2016;64(5):1118-1127.

85. Magalhaes I, et al. Mucosal-associated invariant T cell alterations in obese and type 2 diabetic patients. JClin Invest. 2015;125(4):1752-1762.

86. Gao B, Radaeva S, Park O. Liver natural killer and natural killer $\mathrm{T}$ cells: immunobiology and emerging roles in liver diseases. J Leukoc Biol. 2009;86(3):513-528.

87. Maricic I, et al. Inhibition of type I natural killer $\mathrm{T}$ cells by retinoids or following sulfatide-mediated activation of type II natural killer T cells attenuates alcoholic liver disease in mice. Hepatology. 2015;61(4):1357-1369.

88. Feuerer M, et al. Lean, but not obese, fat is enriched for a unique population of regulatory $\mathrm{T}$ cells that affect metabolic parameters. Nat Med. 2009;15(8):930-939.

89. Wagner NM, et al. Circulating regulatory $\mathrm{T}$ cells are reduced in obesity and may identify subjects at increased metabolic and cardiovascular risk. Obesity (Silver Spring). 2013;21(3):461-468.

90. Salomon B, et al. B7/CD28 costimulation is essential for the homeostasis of the $\mathrm{CD} 4{ }^{+} \mathrm{CD} 25^{+}$ immunoregulatory $\mathrm{T}$ cells that control autoimmune diabetes. Immunity. 2000;12(4):431-440.

91. Poggi M, et al. CD28 deletion improves obesityinduced liver steatosis but increases adiposity in mice. Int JObes (Lond). 2015;39(6):977-985.

92. Chatzigeorgiou A, et al. Dual role of B7 costimulation in obesity-related nonalcoholic steatohepatitis and metabolic dysregulation. Hepatology. 2014;60(4):1196-1210.

93. Bataller R, Brenner DA. Liver fibrosis. JClin Invest. 2005;115(2):209-218.

94. Kisseleva T, et al. Myofibroblasts revert to an inactive phenotype during regression of liver fibrosis. Proc Natl Acad Sci US A. 2012;109(24):9448-9453.

95. She H, Xiong S, Hazra S, Tsukamoto H. Adipogenic transcriptional regulation of hepatic stellate cells. J Biol Chem. 2005;280(6):4959-4967.

96. Blaner WS, et al. Hepatic stellate cell lipid droplets: a specialized lipid droplet for retinoid storage. Biochim Biophys Acta. 2009;1791(6):467-473.

97. Marra F, et al. Ligands of peroxisome prolif- 
erator-activated receptor gamma modulate profibrogenic and proinflammatory actions in hepatic stellate cells. Gastroenterology. 2000;119(2):466-478.

98. Hellerbrand C, Stefanovic B, Giordano F, Burchardt ER, Brenner DA. The role of TGF $\beta 1$ in initiating hepatic stellate cell activation in vivo. JHepatol. 1999;30(1):77-87.

99. Pradere JP, et al. Hepatic macrophages but not dendritic cells contribute to liver fibrosis by promoting the survival of activated hepatic stellate cells in mice. Hepatology. 2013;58(4):1461-1473.

100. Oakley F, et al. Inhibition of inhibitor of $\kappa B$ kinases stimulates hepatic stellate cell apoptosis and accelerated recovery from rat liver fibrosis. Gastroenterology. 2005;128(1):108-120.

101. Miura K, et al. Toll-like receptor 9 promotes steatohepatitis by induction of interleukin- $1 \beta$ in mice. Gastroenterology. 2010;139(1):323-34.e7.

102. Potter JJ, Rennie-Tankesley L, Mezey E. Influence of leptin in the development of hepatic fibrosis produced in mice by Schistosoma mansoni infection and by chronic carbon tetrachloride administration. J Hepatol. 2003;38(3):281-288.

103. Ikejima K, et al. Leptin receptor-mediated signaling regulates hepatic fibrogenesis and remodeling of extracellular matrix in the rat. Gastroenterology. 2002;122(5):1399-1410.

104.Wang J, et al. Kupffer cells mediate leptininduced liver fibrosis. Gastroenterology. 2009;137(2):713-723.

105. Choi SS, et al. Leptin promotes the myofibroblastic phenotype in hepatic stellate cells by activating the hedgehog pathway. J Biol Chem. 2010;285(47):36551-36560.

106. Miyahara T, et al. Peroxisome proliferatoractivated receptors and hepatic stellate cell activation. J Biol Chem. 2000;275(46):35715-35722.

107. De Bleser PJ, Xu G, Rombouts K, Rogiers V, Geerts A. Glutathione levels discriminate between oxidative stress and transforming growth factor $-\beta$ signaling in activated rat hepatic stellate cells. J Biol Chem. 1999;274(48):33881-33887.

108. Paik YH, et al. The nicotinamide adenine dinucleotide phosphate oxidase (NOX) homologues NOX1 and NOX2/gp91(phox) mediate hepatic fibrosis in mice. Hepatology. 2011;53(5):1730-1741.

109. Segal BH, Leto TL, Gallin JI, Malech HL, Holland SM. Genetic, biochemical, and clinical features of chronic granulomatous disease. Medicine (Baltimore). 2000;79(3):170-200.

110.Sancho P, Bertran E, Caja L, Carmona-Cuenca I, Murillo MM, Fabregat I. The inhibition of the epidermal growth factor (EGF) pathway enhances TGF- $\beta$-induced apoptosis in rat hepatoma cells through inducing oxidative stress coincident with a change in the expression pattern of the NADPH oxidases (NOX) isoforms. Biochim Biophys Acta. 2009;1793(2):253-263.

111. Lan T, Kisseleva T, Brenner DA. Deficiency of NOX1 or NOX4 prevents liver inflammation and fibrosis in mice through inhibition of hepatic stellate cell activation. PLoS One. 2015;10(7):e0129743.

112. Bettaieb A, et al. Hepatocyte nicotinamide adenine dinucleotide phosphate reduced oxidase 4 regulates stress signaling, fibrosis, and insulin sensitivity during development of steatohepatitis in mice. Gastroenterology. 2015;149(2):468-80.e10.

113. Paik YH, Schwabe RF, Bataller R, Russo MP, Jobin $\mathrm{C}$, Brenner DA. Toll-like receptor 4 mediates inflammatory signaling by bacterial lipopolysaccharide in human hepatic stellate cells. Hepatology. 2003;37(5):1043-1055.

114. Seki E, et al. TLR4 enhances TGF- $\beta$ signaling and hepatic fibrosis. Nat Med. 2007;13(11):1324-1332.

115. Guo J, et al. Functional linkage of cirrhosispredictive single nucleotide polymorphisms of Toll-like receptor 4 to hepatic stellate cell responses. Hepatology. 2009;49(3):960-968.

116. Onichtchouk D, et al. Silencing of TGF- $\beta$ signalling by the pseudoreceptor BAMBI. Nature. 1999;401(6752):480-485.

117. Czochra P, et al. Liver fibrosis induced by hepatic overexpression of PDGF-B in transgenic mice. JHepatol. 2006;45(3):419-428.

118. Wang L, Johnson EE, Shi HN, Walker WA, Wessling-Resnick M, Cherayil BJ. Attenuated inflammatory responses in hemochromatosis reveal a role for iron in the regulation of macrophage cytokine translation. JImmunol. 2008;181(4):2723-2731.

119. Ryan E, et al. Underdiagnosis of hereditary haemochromatosis: lack of presentation or penetration? Gut. 2002;51(1):108-112.

120. Ruddell RG, et al. Ferritin functions as a proinflammatory cytokine via iron-independent protein kinase $\mathrm{C} \zeta$ /nuclear factor $\mathrm{\kappa B}$-regulated signaling in rat hepatic stellate cells. Hepatology. 2009;49(3):887-900.

121. Bridle KR, Crawford DH, Ramm GA. Identification and characterization of the hepatic stellate cell transferrin receptor. Am J Pathol. 2003;162(5):1661-1667.

122. Gonzalez RS, Gilger MA, Huh WJ, Washington $\mathrm{K}$. The spectrum of histologic findings in hepatic outflow obstruction Arch Pathol Lab Med. [published online ahead of print September 28, 2016]. doi:10.5858/arpa.2015-0388-OA.

123. Bringardner BD, Baran CP, Eubank TD, Marsh $\mathrm{CB}$. The role of inflammation in the pathogenesis of idiopathic pulmonary fibrosis. Antioxid Redox Signal. 2008;10(2):287-301.

124. Ghoghari A, et al. Quantitative determination of saroglitazar, a predominantly PPAR $\alpha$ agonist, in human plasma by a LC-MS/MS method utilizing electrospray ionization in a positive mode [published online ahead of print May 17, 2016]. Biomed Chromatogr. doi:10.1002/bmc.3760.

125. Ratziu V, et al. Elafibranor, an agonist of the peroxisome proliferator-activated receptor- $\alpha$ and $-\delta$, induces resolution of nonalcoholic steatohepatitis without fibrosis worsening. Gastroenterology. 2016;150(5):1147-1159.e5.

126. Ananthanarayanan $M$, Balasubramanian $N$, Makishima M, Mangelsdorf DJ, Suchy FJ. Human bile salt export pump promoter is transactivated by the farnesoid X receptor/bile acid receptor. J Biol Chem. 2001;276(31):28857-28865.

127. Porez G, Prawitt J, Gross B, Staels B. Bile acid receptors as targets for the treatment of dyslipidemia and cardiovascular disease. J Lipid Res. 2012;53(9):1723-1737.

128. Mudaliar S, et al. Efficacy and safety of the farnesoid X receptor agonist obeticholic acid in patients with type 2 diabetes and nonalcoholic fatty liver disease. Gastroenterology. 2013;145(3):574-582.e1.

129. Neuschwander-Tetri BA, et al. Farnesoid X nuclear receptor ligand obeticholic acid for noncirrhotic, non-alcoholic steatohepatitis (FLINT) a multicentre, randomised, placebo-controlled trial. Lancet. 2015;385(9972):956-965.

130. Gilat T, et al. Prevention of diet-induced fatty liver in experimental animals by the oral administration of a fatty acid bile acid conjugate (FABAC). Hepatology. 2003;38(2):436-442.

131. Safadi $\mathrm{R}$, et al. The fatty acid-bile acid conjugate Aramchol reduces liver fat content in patients with nonalcoholic fatty liver disease. Clin Gastroenterol Hepatol. 2014;12(12):2085-2091.e1.

132. Miura K, Yang L, van Rooijen N, Ohnishi H, Seki E. Hepatic recruitment of macrophages promotes nonalcoholic steatohepatitis through CCR2. Am J Physiol Gastrointest Liver Physiol. 2012;302(11):G1310-G1321.

133. Seki E, et al. CCR1 and CCR5 promote hepatic fibrosis in mice. JClin Invest. 2009;119(7):1858-1870.

134. Berres ML, et al. Antagonism of the chemokine Ccl5 ameliorates experimental liver fibrosis in mice. J Clin Invest. 2010;120(11):4129-4140.

135. Friedman S, et al. Efficacy and safety study of cenicriviroc for the treatment of non-alcoholic steatohepatitis in adult subjects with liver fibrosis: CENTAUR Phase $2 \mathrm{~b}$ study design. Contemp Clin Trials. 2016;47:356-365.

136. Pilling D, Buckley CD, Salmon M, Gomer RH. Inhibition of fibrocyte differentiation by serum amyloid P. JImmunol. 2003;171(10):5537-5546.

137. Maharjan AS, Roife D, Brazill D, Gomer RH. Serum amyloid P inhibits granulocyte adhesion. Fibrogenesis Tissue Repair. 2013;6(1):2.

138. Pilling $\mathrm{D}$, et al. Reduction of bleomycin-induced pulmonary fibrosis by serum amyloid P. J Immunol. 2007;179(6):4035-4044.

139. van den Blink B, et al. Recombinant human pentraxin-2 therapy in patients with idiopathic pulmonary fibrosis: safety, pharmacokinetics and exploratory efficacy. Eur Respir J. 2016;47(3):889-897.

140. Ling $\mathrm{H}$, et al. Transforming growth factor $\beta$ neutralization ameliorates pre-existing hepatic fibrosis and reduces cholangiocarcinoma in thioacetamide-treated rats. PLoS One. 2013;8(1):e54499.

141. Lacouture ME, et al. Cutaneous keratoacanthomas/squamous cell carcinomas associated with neutralization of transforming growth factor $\beta$ by the monoclonal antibody fresolimumab (GC1008). Cancer Immunol Immunother. 2015;64(4):437-446.

142. Morris JC, et al. Phase I study of GC1008 (fresolimumab): a human anti-transforming growth factor-beta (TGF $\beta$ ) monoclonal antibody in patients with advanced malignant melanoma or renal cell carcinoma. PLoS One. 2014;9(3):e90353.

143. Trachtman H, et al. A phase 1, single-dose study of fresolimumab, an anti-TGF- $\beta$ antibody, in treatment-resistant primary focal segmental glomerulosclerosis. Kidney Int. 2011;79(11):1236-1243.

144. Rice LM, et al. Fresolimumab treatment decreases biomarkers and improves clinical symptoms 
in systemic sclerosis patients. JClin Invest. 2015;125(7):2795-2807.

145. Koyama Y, et al. Effects of oral intake of hydrogen water on liver fibrogenesis in mice. Hepatol Res. 2014;44(6):663-677.

146.Iwaisako K, et al. Protection from liver fibrosis by a peroxisome proliferator-activated receptor $\delta$ agonist. Proc Natl Acad Sci U S A. 2012;109(21):E1369-E1376.

147. Mato JM, Martinez-Chantar ML, Lu SC. Methionine metabolism and liver disease. Аnnu Rev Nutr. 2008;28:273-293.

148. Pacana T, et al. Dysregulated hepatic methionine metabolism drives homocysteine elevation in diet-induced nonalcoholic fatty liver disease.
PLoS One. 2015;10(8):e0136822.

149. Pradhan M, Shao Y. Clinical grading of intrahepatic cholestasis pregnancy. JNMA J Nepal Med Assoc. 2013;52(190):413-419.

150. Kohjima M, et al. Pathophysiological analysis of primary biliary cirrhosis focusing on choline/phospholipid metabolism. Liver Int. 2015;35(3):1095-1102.

151. Aleynik MK, Lieber CS. Dilinoleoylphosphatidylcholine decreases ethanol-induced cytochrome P4502E1. Biochem Biophys Res Commun. 2001;288(4):1047-1051.

152. Tkachenko P, Maevskaya M, Pavlov A, Komkova I, Pavlov C, Ivashkin V. Prednisolone plus Sadenosil-L-methionine in severe alcoholic hepa- titis. Hepatol Int. 2016;10(6):983-987.

153. Zhang L, et al. Ursodeoxycholic acid and Sadenosylmethionine in the treatment of intrahepatic cholestasis of pregnancy: a multi-centered randomized controlled trial. Eur Rev Med Pharmacol Sci. 2015;19(19):3770-3776.

154. Winer S, et al. Normalization of obesity-associated insulin resistance through immunotherapy. Nat Med. 2009;15(8):921-929.

155. Lalazar G, et al. Oral administration of OKT3 MAb to patients with NASH, promotes regulatory T-cell induction, and alleviates insulin resistance: results of a phase IIa blinded placebo-controlled trial. J Clin Immunol. 2015;35(4):399-407. 